\title{
ORIGINAL
}

\section{UN ESTUDIO DE MINIMIZACIÓN DE COSTES EN LA PRESCRIPCIÓN DE ANTIINFECCIOSOS EN DOS AREAS DE ATENCIÓN PRIMARIA}

\author{
Susana Castán Cameo (1), Florencio Jesús García Latorre (2), Javier Martínez Gorostiaga (2), \\ M. José Sierra Moros (1), Víctor Manuel Solano Bernad (1) y Alfredo Peral Casado (1). \\ (1) Servicio de Medicina Preventiva. Hospital Miguel Servet. Zaragoza. \\ (2) Gerencia de Atención Primaria, Áreas 2 y 5 . Zaragoza.
}

\begin{abstract}
RESUMEN
Fundamento: El gasto farmacéutico es la principal partida de gasto en las áreas de atención primaria. La necesidad de contener el gasto sanitario ha supuesto la búsqueda de alternativas en este campo, una de las cuales seria potenciar una política de prescripción que utilizara la presentación más barata de cada principio activo. El objetivo de este trabajo es evaluar la cantidad que se ahorraría si se optara por prescribir la alternativa más barala en una selección de fármacos antiinfecciosos.

Métodos: Se analizó la prescripción furmacéutica de dos áreas sanitarias a través de la base de datos de facturación de productos farmacéuticos de 1995. Se seleccionaron los monofármacos de acción antiinfecciosa y se buscó para cada principio activo y presentación la alternativa más económica, según el registro facilitado por el Ministerio de Sanidad; realizando un análisis de minimización de costes. No se consideraron los costes de implementación de dicha políticá de prescripción.

Resultados: El gasto farmacéutico total en las áreas fue de 8.547 millones en 1995 . Fl gasto en los antiinfecciosos seleccionados fue de 522 millones $(6,1 \%$ del total $)$. El ahorro global estimado fue del $7.63 \%(39.901 .778$ ptas. $)$. Dicho ahorro se concentró en los subgrupos: penicilinas, quinolonas, cefalosporinas y macrólidos. El $75 \%$ del ahorro potencial identificado se hubiera conseguido con la prescripción de la alternativa más barata de 2 principios activos: amoxicilina y ciprofloxacino.

Conclusiones: El estudio muestra las posibilidades de contención del gasto en nuestro medio y ofrece una base para la actuación en este sentido. Sería conveniente impulsar la información y formación de prescriptores y dispensadores para potenciar el uso de la alternativa más económica de cada medicamento que se prescriba, especialmente en aquellos casos en que haya importantes márgenes para el ahırro.

Palabras clave: Antiinfecciosos. Análivis de costes. Costes farmacéuticos. Eficiencia, Atención Primaria.
\end{abstract}

Correspondencia:

Florencio J. García Latorre

Dirección Provincial de INSALUD

Prolongación $\mathrm{C} / \mathrm{San}$ Jorge $\mathrm{s} / \mathrm{n}$

22003 HUESCA

Teléfono: $974 / 211600$

Fax: 974/212126

\section{ABSTRACT}

\section{A Study to Minimize the Cost of Prescribing Anti-infective Drugs in two Areas of Primary Health Care}

Background: Pharmaceutical costs are the main cost item in basic health care. The need to contain health care expenditure has led to the search for alternatives in this area, one of which would be to foster a prescription policy which uses the cheapest presentation for each active principle. The aim of this study was to evaluate the amount which would he saved hy prescrihing the cheapest alternative from a selection of anti-infective drugs.

Methods: Pharmacy prescriptions in two different health areas were analyzed using the database on turnover of pharmaceutical products for 1995. Single principle drugs with anti-irfective action were selected, and for each active principle and presentation the most economic alternative was sought using the records provided by the Ministry of Health and a cost minimisation analysis was undertaken. The cost of applying this prescription policy was not considered.

Results: Total pharmaceutical expenditure in the areas was pesetas $8.547 \mathrm{bn}$ in 1995. Expenditure on selected anti-infective drugs was pesetas 522 million $(6.1 \%$ of the total). The overall saving estimated was $7.63 \%$ (pesetas $39,901,778$ ). This saving was centred on the following subgroups: penicillins, quinolones, cephalosporins and macrolides. Of potential savings identified, $75 \%$ would be achieved by prescribing the cheaper altemative of 2 active principles: amoxacillin and cyprofloxacine.

Conclusions: The study shows the possibility of containing expenditure in our area and offers a basis for action in this direction. It would be advisable to increase information and training for prescribers and dispensers in order to stimulate the use of the most economical alternative of each medicament prescribed, especially in cases in which there are significant margins to be saved.

Key words: Anti-infective drugs. Cost analysis. Pharmaceutical costs. Efficacy, Basic health care. 


\section{INTRODUCCIÓN}

El gasto farmacéutico representa aproximadamente el $20 \%$ del presupuesto total destinado a la asistencia sanitaria por el Sistema Nacional de Salud ${ }^{1,2}$. El $90 \%$ del consumo de medicamentos corresponde a prescripciones realizadas por los facultativos de atención primaria ${ }^{3}$, siendo para las Gerencias de este nivel asistencial la mayor partida de gasto ${ }^{4}$.

Su crecimiento en los últimos años se debe fundamentalmente al aumento del precio medio por receta y al aumento del número de prescripciones a pensionistas, ya que el número de recetas se ha mantenido relativamente estable ${ }^{3,5}$. La aparición de nuevas moléculas en el mercado (con un elevado precio) y la promoción de la industria farmacéutica sobre los prescriptores se han postulado, así mismo, como factores vinculados al aumento del gasto.

En estas circunstancias, se han generado numerosos estudios ${ }^{3-10}$ sobre los factores condicionantes de la prescripción, especialmente tras la puesta en marcha de la política de Uso Racional del Medicamento (URM), cuyos objetivos se centran en la contención del gasto manteniendo la calidad en la prescripción. Una de las alternativas que se plantean es la disminución del precio medio por receta mediante la prescripción de la marca registrada más barata existente en el mercado, sin detrimento de la calidad terapéutica. En este estudio se ha analizado dicha propuesta para un grupo determinado de fármacos, los antiinfecciosos.

La patología infecciosa bacteriana es un problema de consulta frecuente en atención primaria ${ }^{8.11-13}$. La prescripción de antibióticos supone en este nivel asistencial alrededor de un $15 \%$ sobre el total, porcentaje muy superior al de otros países europeos (Italia $9,5 \%$, Francia $7,6 \%$ e Inglaterra $9,8 \%)^{7,8}$.

El objetivo de este estudio ha sido cuantificar el gasto farmacéutico debido a la prescripción de antiinfecciosos en las áreas 2 y 5 de atención primaria de Zaragoza durante 1995. Así mismo, se ha realizado un análisis de minimización de costes comparando dicho consumo con la prescripción de la presentación de menor coste del mismo principio activo al objeto de conocer el margen de ahorro que se obtendría si dicha política estuviese implementada.

\section{MATERIAL Y MÉTODOS}

Los datos sobre las prescripciones realizadas y dispensadas en las Oficinas de Farmacia durante el año 1995 se obtuvieron a partir del registro informatizado de facturación de recetas médicas del Colegio Oficial de Farmacéuticos procesado por la gerencia de atención primaria de las áreas 2 y 5 del Instituto Nacional de la Salud (Insalud) en Zaragoza. Para cada una de las especialidades farmacéuticas se consideraron las siguientes variables: código nacional, grupo terapéutico, consumo (expresado en número de envases y número de recetas) y precio de venta al público. Dicho consumo se obtuvo de forma global y desglosado para activos y pensionistas.

Se seleccionaron las especialidades farmacéuticas que contenían un solo principio activo antiinfeccioso de los incluidos en los grupos terapéuticos J01 (antibióticos sistémicos) y J03 (quimioterápicos sistémicos) de la clasificación anatómico-terapéutica de medicamentos ${ }^{14}$ (A.T.C.) adoptada en España desde 1989, excluyendo las presentaciones calificadas como de diagnóstico hospitalario.

Los precios oficiales de las especialidades farmacéuticas se tomaron del Nomenclátor facilitado por el Servicio de Banco de Datos de Medicamentos de la Dirección General de Farmacia y Productos Sanitarios del Ministerio de Sanidad y Consumo. En esta base de datos de medicamentos, cada especialidad farmacéutica viene descrita por los siguientes campos: código nacional, grupo terapéutico, 
marca registrada y presentación, composición, laboratorio fabricante, precio de venta al público y situación de comercialización. De este listado se obtuvo la especialidad de menor precio entre las posibles alternativas.

A partir del consumo por receta de estos antiinfecciosos, se ha realizado un análisis de minimización de costes para cuantificar el ahorro teórico que supondría la prescripción del mismo principio activo pero de la marca registrada más barata y con la misma presentación, sin incluir los costes necesarios para la implementación de dicha política de prescripción.

El análisis de minimización de costes es una forma de evaluación aplicable cuando se valoran alternativas terapéuticas a las que se presume el mismo resultado sanitario, la misma eficacia farmacológica y los mismos efectos secundarios, es decir, se asume que presentan la misma efectividad terapéutica $^{15-18}$. De este modo, basta con la comparación entre los costes de cada una de las opciones para identificar la menos gravosa.

En este caso, se ha asumido que presentaciones con la misma composición y dosis tienen una eficacia terapéutica equivalente. Así, se ha considerado que dos especialidades farmacéuticas son equivalentes desde el punto de vista farmacológico si contienen el mismo principio activo en cuanto a dosis, vía de administración y número de unidades en la presentación. En la vía de administración oral.se incluyen las presentaciones sobres, comprimidos y cápsulas, considerando por separado las presentaciones en soluciones o jarabes orales. Esta clasificación se ha utili- zado tanto para los fármacos prescritos como para la elección de la alternativa más barata.

En este trabajo no se ha valorado si la indicación del tratamiento es correcta, ni tampoco el grado de cumplimiento terapéutico de los pacientes.

La población asignada a las áreas 2 y 5 de Zaragoza en 1995 fue de 532.457 personas (384.657 activos y 147.800 pensionistas). El número de zonas básicas de salud en el período estudiado era de 35 , repartidas según modelo de atención en 19 equipos de atención primaria urbanos, 4 equipos rurales, 4 consultorios de modelo tradicional urbanos y 8 zonas atendidas por modelo tradicional rural. El número total de facultativos que trabajaban en las árcas era de 409 (283 médicos generales, 67 pediatras, 37 facultativos del servicio de urgencias y 22 médicos de Unidades de Apoyo).

\section{RESULTADOS}

\section{Análisis General}

Durante 1995, el gasto farmacéutico total en las áreas, sin incluir la aportación de los asegurados, superó los 8.500 millones de pesetas, correspondiente a la prescripción de algo más de 6 millones de recetas. De estas cantidades, el grupo terapéutico J supuso el $12,15 \%$ del gasto y el $10,21 \%$ del total de recetas. Por su parte, los AS (antiinfecciosos seleccionados) representaron el $6,1 \%$ del gasto total y el $4,8 \%$ de las prescripciones a través de receta médica. En la tabla 1 se ha reflejado información más pormenorizada sobre estos aspectos.

Tabla 1

Gasto farmaceútico, recetas prescritas y gasto medio por asegurado y por receta en 1995

\begin{tabular}{|lcccccc|}
\hline & $\begin{array}{c}\text { Gasto } \\
\text { fammáutico } \\
\text { (pesetas) }\end{array}$ & $\begin{array}{c}\% \\
\text { sobre total }\end{array}$ & $\begin{array}{c}N^{\circ} \text { de } \\
\text { recetas }\end{array}$ & $\begin{array}{c}\% \\
\text { sobre total }\end{array}$ & $\begin{array}{c}\text { Gasto medio } \\
\text { por receta } \\
\text { (pesetas) }\end{array}$ & $\begin{array}{c}\text { Gasto medio por } \\
\text { asegurado } \\
\text { (pesetas) }\end{array}$ \\
\hline Total prescripciones & 8.547 .421 .675 & 100 & 6.067 .373 & 100 & 1.404 & 16.053 \\
Total antiinfecciosos & 1.038 .511 .173 & 12,1 & 619.479 & 10.21 & 1.676 & 1.950 \\
Total principios seleccionados & 522.624 .670 & 6,1 & 292.498 & 4.8 & 1.786 & 981 \\
\hline
\end{tabular}


El $62,7 \%$ de las recetas de los AS han sido prescritas sobre receta de activos y el $37,3 \%$ en receta de pensionista. A su vez, los AS, supusieron un 9,3\% del total de prescripciones realizadas para población activa y un $2,6 \%$ para pensionistas.

Estos dos grupos de población presentan notables diferencias en algunos parámetros. El gasto medio por receta de los AS fue de 1.107 pesetas para la población activa y de 2.931 pesetas para pensionistas. Del mismo modo, el gasto medio por persona para los AS fue de 528 ptas. en activos y 2.162,5 pesetas. en pensionistas. Por último, la relación recetas por persona fue de 0,58 en activos y 0,74 en pensionistas.

La base de datos empleada contenía un total de 856 presentaciones existentes en el mercado de los AS. Sin embargo, sólo se registró consumo de 447 de ellas en el año estudiado, es decir, del $52 \%$.

\section{Análisis por subgrupos terapéuticos}

El consumo según los subgrupos de antiinfecciosos expresado en porcentaje de recetas prescritas para activos y pensionistas se describe en la tabla 2.
El mayor porcentaje de consumo por receta para la población activa fue en los subgrupos Penicilinas, Macrólidos y Cefalosporinas; en población pensionista, en los anteriores, además de las Quinolonas.

El porcentaje de gasto que ha representado cada uno de los subgrupos se expresa en la figura 1. El mayor gasto en población activa ha sido en Macrólidos $(35,57 \%)$, Cefalosporinas $(27,35 \%)$ y Penicilinas de amplio espectro $(20,79 \%)$, y para población pensionista, los mismos subgrupos con un $26,91 \%, 35,47 \%$ y $9,52 \%$ respectivamente, añadiendo además Quinolonas $(22,73 \%)$, subgrupos que en ambas poblaciones coinciden con los de mayor prescripción.

\section{Análisis por principios activos}

El principio activo antibiótico más recetado ha resultado ser la amoxicilina, especialmente en la presentación de $500 \mathrm{mg}, 16$ sobres, seguido del principio activo cefonicid lgr inyectable. Las 10 presentaciones de monofármacos más recetados y consumidos en 1995 se encuentran relacionadas en la figura 2.

Tabla 2

Porcentaje de recetas prescritas por subgrupos de antiinfecciosos

\begin{tabular}{|c|c|c|c|c|}
\hline & \multicolumn{2}{|c|}{ ACTIVOS } & \multicolumn{2}{|c|}{ PENSIONISTAS } \\
\hline & $N .^{\circ}$ recetas & $\%$ & $N .^{\circ}$ recetas & $\%$ \\
\hline Tetraciclinas & 5.902 & 3,22 & 2.186 & 2 \\
\hline Penicilinas amplio espectro & 79.023 & 43,1 & 30.911 & 28.35 \\
\hline Cefalosporinas & 30.168 & 16,44 & 22.146 & 20,31 \\
\hline Macrólidos y similares & 52.688 & 28,72 & 33.532 & 30,75 \\
\hline Aminoglucósidos & 492 & 0,27 & 874 & 0,8 \\
\hline Rifampicinas y afines & 459 & 0,25 & 192 & 0,18 \\
\hline Sulfamidas & 238 & 0,13 & 227 & 0,21 \\
\hline Fosfomicina & 1.460 & 0,8 & 2.140 & 1,96 \\
\hline Quinolonas & 6.098 & 3,32 & 13.735 & 12,6 \\
\hline Midecamicina & 6.903 & 3,76 & 2.975 & 2,73 \\
\hline Otros * & 12 & 0 & 132 & 0,12 \\
\hline TOTAL & 183.448 & 100 & 109.050 & 100 \\
\hline
\end{tabular}

* Se refiere a los subgrupos: Cloranfenicol, Aztreonam y Colistina. 
Figura 1

Porcentaje de gasto en antibióticos según los grupos seleccionados

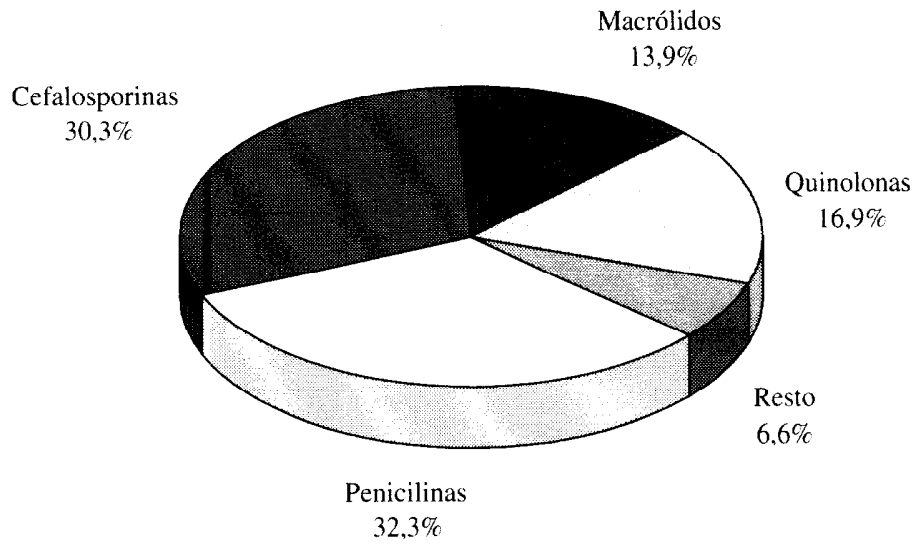

Figura 2

Presentaciones farmacológicas más consumidas (en número de recetas)

Amoxicilina 500 mg 16 sobres

Cefonicid 1 gr. inyectable

Claritromicina $250 \mathrm{mg} .12$ compr.

Eritromicina $50(0 \mathrm{mg} 12$ sobres

Amoxicilina lgr. 12 comprimidos

Amoxicilina $750 \mathrm{mg} 12$ tabletas

Amoxicilina $500 \mathrm{mng} 12$ cápsulas

Amoxicilina $250 \mathrm{mg} 120 \mathrm{ml}$. sus.

Minociclina $100 \mathrm{mg} 12$ cápsulas

Eritromicina 1 gr. 12 sobres

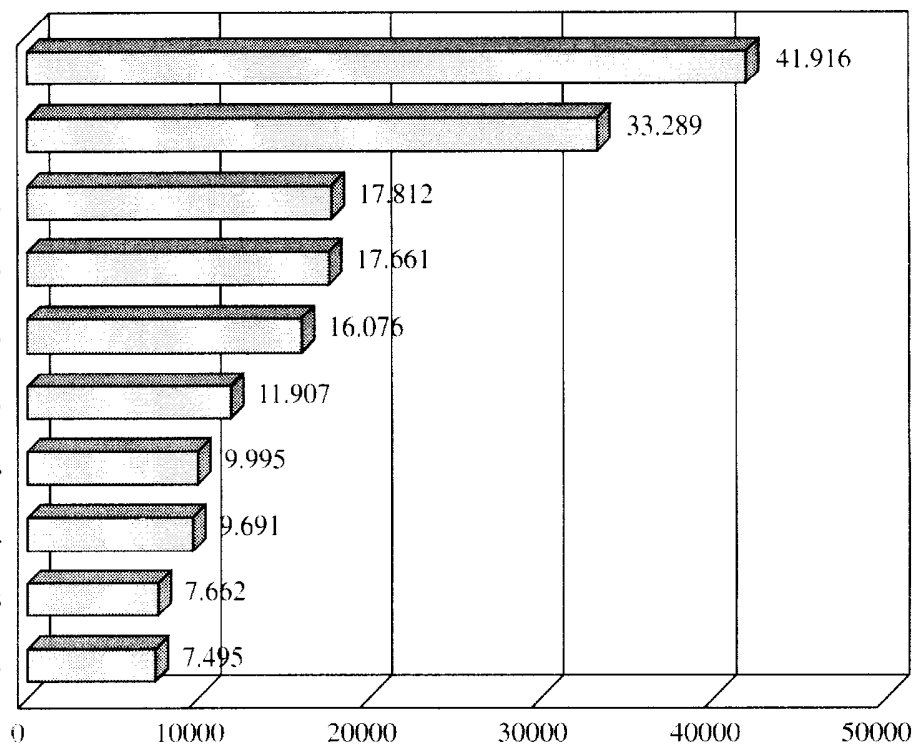




\section{Minimización de costes}

El ahorro medio global que hubiera supuesto la prescripción del mismo principio activo pero de la marca registrada más barata es del 7,63\%.

En la tabla 3 se han agrupado los resultados por subgrupos. En dicha tabla, el ahorro estimado para cada uno de ellos, se expresa primero mediante la cantidad de pesetas ahorradas (diferencia entre el gasto real en 1995 en ese subgrupo y el gasto estimado con la elección de la marca registrada más barata), y segundo, en el porcentaje que dicha cantidad representa dentro de cada subgrupo.

Para la población activa destaca que el ahorro estimado en el subgrupo Penicilinas de amplio espectro es de un $23 \%$ y de un $15 \%$ en Quinolonas. En los otros subgrupos de elevado consumo en la población activa, como son Macrólidos y Cefalosporinas el ahorro que se ha estimado es inferior al 3\%; sin embargo, en Aminoglicósidos, con un porcentaje de ahorro importante $(14 \%)$ representa una cantidad poco relevante debido a su baja prescripción.
Para la población pensionista, también el máximo ahorro estimado ha sido en Penicilinas de amplio espectro con un $23 \%$ y de un $16 \%$ en Quinolonas. En esta población ocurre, como en la población activa, que Cefalosporinas y Macrólidos, en los que se estima un porcentaje de ahorro bajo en su grupo, representa cantidades relativamente importantes. Contrariamente, en el caso del cloramfenicol el ahorro estimado aún siendo casi de un $24 \%$ representa una cantidad mínima. Como en el caso de la población acti$\mathrm{va}$, en este colectivo existen subgrupos en los que no se obtiene ahorro, ya que es coincidente el gasto real con el gasto estimado al no existir otra alternativa más barata.

Si se considera la contribución de cada subgrupo de antiinfecciosos al ahorro total se obtiene la siguiente distribución: el $42,4 \%$ del ahorro correspondería a penicilinas de amplio espectro, el $35,4 \%$ a quinolonas, el $10,8 \%$ a cefalosporinas, el $10 \%$ a macrólidos y similares, $\mathrm{y}$ un pequeño porcentaje $(0,54 \%)$ en tetraciclinas, fosfomicina y aminoglucósidos.

El ahorro estimado en pesetas para cada principio activo se expresa en la tabla 4. Teniendo en cuenta la prescripción de la al-

Tabla 3

Ahorro estimado con la elección de la alternativa más barata por subgrupos

\begin{tabular}{|lcrrrrrr|}
\hline & \multicolumn{2}{c}{ ACTIVOS } & \multicolumn{2}{c|}{ PENSIONISTAS } & \multicolumn{2}{c|}{ TOTAL } \\
\cline { 2 - 7 } & Ahorro en ptas. & \% ahorro & Ahorro en ptas. & \% ahorro & Ahorro en ptas. & \% Ahorro \\
\hline Tetraciclinas & 66.921 & 1,65 & 75.069 & 3,63 & 141.990 & 2,32 \\
Cloranfenicol & 21 & 4,48 & 496 & 23,87 & 517 & 20,23 \\
Penicilinas amplio espectro & $9.799 .909,6$ & 23,22 & 7.099 .718 & 23,32 & 16.899 .627 & 23,26 \\
Cefalosporinas & $1.441 .338,8$ & 2,60 & 2.871 .130 & 2,53 & 4.312 .469 & 2,55 \\
Macrólidos y similares & $1.760 .192,8$ & 2,44 & 2.220 .417 & 2,58 & 3.980 .610 & 2,52 \\
Aminoglucósidos & 88.416 & 13,98 & 308.072 & 16,03 & 11.625 & 15,52 \\
Rifampicinas y afines & 8.987 & 1,88 & 2.639 & 0,61 & 0 & 1,28 \\
Fosfomicina & 15.695 & 1,28 & 26.980 & 0,82 & 42.675 & 0,94 \\
Quinolonas & $2.430 .028,6$ & 15,45 & 11.685 .747 & 16,09 & $14.115 .775,8$ & 15,97 \\
Otros * & 0 & 0 & 0 & 0 & 0 & 0 \\
TOTAL & & & & & 0 \\
\end{tabular}

* Otros hace referencia a los subgrupos: Aztreonam, Colistina, Sulfamidas y Midecamicina. 
Tabla 4

Ahorro estimado con la elección de la alternativa más barata según principios activos

\begin{tabular}{|lcc|}
\hline & \multicolumn{2}{c|}{ AHORRO ESTIMADO } \\
\cline { 2 - 3 } & Importe ptas. $\left.{ }^{*}{ }^{*}\right)$ & \% Ahorro \\
\hline Amoxicilina & 16.389 .083 & 25 \\
Ciprofloxacino & 12.818 .536 & 16,3 \\
Eritromicina & 2.822 .440 & 11,6 \\
Cefuroxima & 1.595 .558 & 3.5 \\
Ofloxacino & 1.230 .061 & 15,1 \\
Cefonicid & 1.182 .115 & 2 \\
Claritromicina & 859.555 & 1,4 \\
Cefaclor & 701.890 & 5,3 \\
Otros & 2.302 .540 & $(* *)$ \\
\hline TOTAL & 39.901 .778 & 100 \\
\hline
\end{tabular}

(*) La diferencia en el gasto entre las dos alternativas, es decir, el gasto real en 1995 y el gasto que se hubiera producido con la elección de la alternativa más barata para cada principio activo

${ }^{(* *)}$ No se ha desglosado el porcentaje de ahorro en cada uno de los principios activos restantes de este subgrupo.

ternativa más barata de únicamente 8 principios activos se ahorraría el $94,2 \%$ de la cantidad señalada anteriormente como gasto total potencialmente evitable. Es decir, con la prescripción de las presentaciones más baratas en la amoxicilina se ahorraría el $41,1 \%$ del total, en ciprofloxacino el $32,12 \%$, en la eritromicina el $7,1 \%$, en cefuroxima un $4 \%$, ofloxacino el $3,1 \%$, cefonicid el $2,9 \%$, claritromicina el $2,2 \%$, cefaclor el $1,8 \%$ y con todo el resto de principios activos se ahorraría sólo el $5,8 \%$.

El ahorro estimado con la elección de la alternativa más barata presenta diferentes niveles en dependencia del grupo de antiinfecciosos del que se trate. Así, existen subgrupos terapéuticos en los que, con el consumo de 1995, se hubiera ahorrado en promedio por cada receta prescrita aproximadamente 400 pesetas, como es el caso del subgrupo de quinolonas para la población activa.

En la población pensionista el mayor ahorro por receta se obtiene en quinolonas, alcanzando la cifra de 850 ptas. y Amino- glucósidos con un gasto evitable por prescripción de 352 ptas.

El $40,6 \%$ de las recetas no son candidatas a ningún tipo de ahorro, es decir, no existe alternativa más económica (tabla 5).

Tabla 5

Porcentaje de recetas susceptibles de ahorro; ahorro medio en activos y pensionistas

\begin{tabular}{|c|c|c|}
\hline & Activos & Pensionistas \\
\hline $\begin{array}{l}\text { Porcentaje recetas suscepti- } \\
\text { bles de ahorro }\end{array}$ & $58,5 \%$ & $60,9 \%$ \\
\hline Ahorro medio & 145,6 ptas. & 365,7 ptas. \\
\hline
\end{tabular}

\section{DISCUSION}

La limitación de recursos disponibles para el funcionamiento de los servicios de salud está generando un progresivo aumento en la producción científica en relación con la economía de la salud. Por el momento en nuestro país no son muchos los trabajos publicados en estc árca de conocimiento y son relativamente escasos los dedicados a la farmacoeconomía ${ }^{1,35.6,69,19-21}$.

La utilización de recursos públicos en una determinada actividad impide su uso en otras que también serían de utilidad para la mcjora de la salud de la población. En este caso, la partida dedicada a farmacia absorbe una quinta parte del presupuesto total de sistema sanitario $^{2}$ por lo que su importancia es manifiesta. En los últimos años se han venido ensayando múltiples medidas de contención $^{22}$, como la exclusión de determinados productos de la financiación pública a raíz del RD 83/93 sobre financiación de medicamento $^{23}$ o la reducción de márgenes comerciales a las oficinas de farmacia. Fuera de nuestras fronteras, otras opciones utilizadas para contener costes han sido el establecimicnto de precios fijos financiados por el medio público, de modo que para fármacos con un coste superior, la diferencia corre a cargo del usuario (Alemania, Holanda), o 
una amplia promoción de la prescripción de genéricos ${ }^{24}$.

En otros países los precios de estos medicamentos genéricos resultan más baratos que los de marca. Debido a que en España hasta 1992 ha estado vigente la patente de procedimiento, en el mercado español existen "falsos genéricos", es decir, medicamentos con denominación genérica que son copias de otros similares y cuyo período de patente no ha finalizado. En algunos casos, el precio de los medicamentos con marcas de fantasía es inferior al de los "genéricos" existentes en nuestro país, como se ha podido constatar mediante la revisión realizada, por lo que el debate sobre la utilización o no de los productos así denominados, como modo de disminuir el gasto, debe ir más allá de la simple nomenclatura.

La recomendación de una política de genéricos podría entrar en contradicción con el principio de libertad de prescripción pero, sin embargo, parece lógico pensar que en una situación de contención del gasto sanitario ésta puede ser una vía lícita para disminuir el gasto sin perjudicar la calidad de la prestación farmacéutica de cara al ciudadano. En general, el consumo de genéricos en España no supera el $1 \%{ }^{25}$; en terapia antiinfecciosa, se encuentran genéricos para un reducido número de principios activos.

Es obvio que la implementación de una nueva política de prescripción requiere de intervenciones que generarían cl consiguiente consumo de recursos, que en este trabajo no se han tenido en cuenta. Sin embargo, esta minimización de costes puede ser útil para valorar los beneficios (ahorro teórico) que esa política generaría y contrapesarlos con los costes de su puesta en marcha y así valorar la factibilidad de la intervención.

En este estudio se ha contado con que distintas presentaciones comerciales, conteniendo la misma cantidad de principio activo y administrables por la misma vía, presentan una efectividad equiparable. Se podría criticar esta asunción señalando que, posiblemente, los productos no son bioequivalentes, que los excipientes pueden dar lugar a efectos distintos sobre la farmacocinética del producto o bien, que por este mismo motivo, pueden generar efectos secundarios diferentes. Debido a que lo sustancial es el principio activo, estas cuestiones no parecen ser de excesiva relevancia de cara a la validez del estudio. La aplicación de la minimización de costes sobre monofárma$\cos$, sin incluir combinaciones, facilita el objetivo trazado.

Desde las áreas de atención primaria se ha venido trabajando en los últimos años en la elaboración de guías farmacoterapéuticas para facilitar la toma de decisiones en el tratamiento de una determinada patología. El coste ha sido uno de los elementos de juicio aportados en dichas guías. El conocimiento de costes comparativos entre productos parece modular de algún modo los hábitos de prescripción ${ }^{19,26}$. Esta medida puede tener efectos a más largo plazo que cualquier otra intervención, al ser los profesionales los principales implicados en la prescripción.

El gasto farmacéutico está estrechamente vinculado al consumo de las diferentes moléculas y su política de utilización. La dispensación de antibióticos en España, según algunos autores ${ }^{8}$, representa un porcentaje mayor que en otros países europeos. En concreto, en las áreas del estudio ha representado un $12,15 \%$ sobre el gasto farmacéutico total.

El perfil del consumo de antiinfecciosos obtenido coincide con el de otros estudios ${ }^{7.26}$ siendo, en este trabajo también, el subgrupo de penicilinas de amplio espectro el más importante sobre todo para la población activa. En un estudio realizado en un área limítro$\mathrm{fe}^{27}$, también se ha observado la coincidencia en los subgrupos terapéuticos más utilizados: penicilinas, macrólidos, tetraciclinas y cefalosporinas. La literatura recoge el importante aumento de las cefalosporinas y quinolonas en atención primaria. quizá motivado por el desarrollo de nuevas moléculas ; parece de interés, la monitorización del uso de algunos de estos antimicrobianos so- 
bre todo teniendo en cuenta el creciente aumento de las resistencias bacterianas como problema importante de salud pública" ${ }^{\prime \prime}$.

De los tres subgrupos de antiinfecciosos más prescritos, la mayor reducción en porcentaje de ahorro corresponde a las penicilinas. En gasto evitable por receta, sería el subgrupo de las quinolonas el de mayor relevancia. Estas dos informaciones marcan un camino a seguir en la información a los profesionales, del mismo modo que la constatación de que la intervención sobre tan sólo 8 principios activos puede suponer el $94 \%$ del ahorro total en los monofármacos seleccionados, y casi el $75 \%$ con únicamente dos principios (amoxicilina y ciprofloxacino).

Las medidas de contención del gasto en farmacia dependen de múltiples factores, cada uno de ellos con distinta relevancia y posibilidades de intervención. Los estudios de farmacoeconomía, así como los referidos a idoneidad de la prescripción y al cumplimiento del tratamiento pueden ser de gran ayuda en la toma de decisiones sobre las medidas más adecuadas para mejorar la eficiencia en la prestación farmacéutica. Su éxito pasa por su capacidad de generar una responsabilidad compartida entre responsables de la información, prescriptores, dispensadores y usuarios $1,3,5,8,9,28,29$.

\section{BIBLIOGRAFÍA}

1. 'Arroyo MP, Cano R, Ansorena J, Celay F, Cortés F, Estremera V. Prescripción delegada por especialistas en atención primaria. Aten Primaria 1995; 16 : $538-44$

2. Temes Montes JL, Gil Redrado J. Sistema Nacional de Salud. Madrid: McGraw Hill-Interamericana; 1997.

3. Azagra R. ¿Podemos disminuir los costes de farmacia? Aten Primaria 1993; 3: 117-8.

4. García Latorre FJ, Dolsac Espinosa JI, Cebrián Martín C, Lorente Valero F, Bastarós García JC. Indicadores económico-asistenciales en dos áreas sanitarias: el coste del "producto consulta" en equipos de atención primaria. Aten Primaria 1994; 3: 655-60.
5. Arnau JM. Medidas de contención del gasto farmacéutico e indicadores de calidad en el uso de los medicamentos: iprescribir menos o prescribir mejor?. Aten Primaria 1994; 4: 155-8.

6. Jiménez Puente A, Ordóñez Martí-Aguilar MV, Córdoba Doña JA, Fernández Gómez MA. Factores relacionados con el gasto y la calidad de la prescripción en atención primaria. Aten Primaria 1995; 16: 131-6.

7. Baena Jiménez M, Montero Pérez FJ, Barco Enríquez MC, Calderón de la Barca JM. La prescripción antibiótica en Atención Primaria. Aten Primaria 1990; 8: 589-90.

8. Uso de antimicrobianos en Atención Primaria. Aten Primaria 1986; 3: 112.

9. Lomeña Villalobos JA, Ceballos Vacas M, Medina Arteaga MT, Mediavilla Cordero E, Sarmiento Jiménez F, Hernández Grande JL. Gasto farmacéutico en atención primaria según el origen de las prescripciones. Aten Primaria 1996; 1: 75-8.

10. Espigares Arroyo M, Montes Salas G, Altimiras Roset J, Iglesias Sánchez JM, Brioso Jerez F. Factores predictivos de la prescripción farmacéutica: perfil del médico hiperprescriptor. Gac Sanit 1994; 8: $25-9$.

11. Pérez Sáenz JL, García MD. Antibióticos y resistencia bacteriana. Aten Primaria. FALTAN DATOS.

12. Mendive Arbeloa JM, Vinyoles Bargallo E, Mata Cases M, Altaba Barcelo AM, García Ruiz F, Salvador González B, Solsona Díaz L, Wennberg Rutllant MP. Patología infecciosa en atención primaria. Aten Primaria 1996; 17: 90-4.

13. Juncosa S, Ledesma A, Carvajal JA. Calidad del tratamiento antibiótico en la atención primaria de la comarca de Osona (Barcelona). Med Clin (Barc) 1994; 103: 252-257.

14. Orden del 13-10-1989 sobre la clasificación anatómica de medicamentos. BOE núm 257 de $26 / 10 / 1989$.

15. Badía X, Rovira J. Evaluación económica de medicamentos. Madrid: Luzán 5; 1994.

16. Sacristán JA, Badía X, Rovira J. Farmacoeconomía: Evaluación económica de medicamentos. Madrid: Editores Médicos; 1995.

17. Drummond MF, Stoddart GL, Torrance GW. Métodos para la evaluación económica de los programas de atención de la Salud. Madrid: Díaz de Santos; 1991.

18. Rovira $\mathbf{J}$ (Coordinador). Glosario de términos y conceptos de uso frecuente en la evaluación cco- 
nómica de medicamentos y programas sanitarios. Barcelona: Soikos; 1996.

19. Ansa Erice Y, Agudo Pascual C, Artazcoz Sanz M, Carnicero Giménez de Azcárate J. Utilidad de la prescripción de genéricos en la contención del gasto en prestación farmacéutica. Análisis en la Comunidad Foral de Navarra. Aten Primaria 1996; 17: 411-3.

20. Llor C, Vila A, Gutiérrez M C, Alvarez M, Villalón M. Medicación antihipertensiva: ¿En cuánto podría reducirse el gasto farmacéutico si se prescribiera la especialidad más barata?. Libro de ponencias XIV Congreso Nacional de Medicina Familiar y Comunitaria. Madrid; 1994; 198.

21. Benavent Areu J, Bordas Julve JM, Casajuana Brunet J, Romea Lecumberri S. Asignación del presupuesto de farmacia a los centros de salud. Aten Primaria 1996; 3: 116-21.

22. Sociedad Española de Salud Pública y Administración Sanitaria. La Salud y el sistema sanitario en España: Informe SESPAS 1995. Barcelona: SG Editores; 1995.

23. Real Decreto $83 / 1993$ de 22 de enero por el que se regula la selección de los medicamentos a efec tos de su financiación por el Sistema Nacional de Salud. BOE núm 43, de 19/2/1993.

24. Limón C. Prestaciones Sanitarias en los países de la Comunidad Europca. Madrid: Ministerio de Sanidad y Consumo; 1994.

25. Plata AM. Medicamentos genéricos. Avifarma $1995 ; 1(10): 1-8$.

26. Roberts SJ, Bateman DN, Smith JM. Prescribing behaviour in general practice: the impact of promoting therapeutically equivalent cheaper medicines. Br J Gen Prac 1997; 47:13-8.

27. Goldaracena Tanco M, Aza Pascual-Salcedo M, Bárcena Caamaño M, Fustero Fernández MV. Consumo extrahospitalario de antiinfecciosos en dosis diaria definida por mil habitantes y dia. Aten Primaria 1996; 18: 357-61.

28. Guerra Aguirre FJ, Crespo Sánchez-Eznarriaga B. Prescripción de genéricos. Aten Primaria 1994; 5: 223-4.

29. Casado Zuriguel Y. Intervención para mejorar la calidad de prescripción de antibióticos en una área básica de salud. Aten Primaria 1993; 1:53-5. 